\title{
Outcomes over the first two years of treatment with mepolizumab in severe asthma
}

Copyright @The authors 2021.
This version is distributed under
the terms of the Creative
Commons Attribution
Non-Commercial Licence 4.0.
For commercial reproduction
rights and permissions contact
permissions@ersnet.org
Received: 7 May 2021
Accepted: 26 Sept 2021 \\ To the Editor: \\ Mepolizumab was the first anti-interleukin-5 (anti-IL-5) monoclonal antibody licensed for the treatment of \\ severe asthma, recommended as add-on treatment in patients with frequent exacerbations or requiring \\ maintenance oral corticosteroids (OCS) despite optimal inhaled therapy [1, 2]. In clinical trials it reduced \\ exacerbation rates and OCS use, and improved lung function, asthma control and health-related quality of \\ life over the first year of treatment [3-6]. Current guidance states that anti-IL-5 biologics should be \\ continued long term if benefit is demonstrated over the first year, yet little is known about whether initial \\ responses are sustained, or whether they can be predicted by clinical characteristics. \\ We report an analysis of data collected over the first 2 years of treatment in patients who had initially \\ responded to mepolizumab (by halving their OCS requirement and/or exacerbation rate over the first \\ 12 months of treatment). We aimed to investigate: rates of continued response (sustained responders) and \\ failure (late failures) beyond month 12; reasons for late failure; predictors of sustained response in clinical \\ parameters at mepolizumab initiation and at month 12; and outcomes in sustained responders. \\ Clinical data were collected from patients with severe asthma attending the regional severe asthma service \\ between May 2017 and June 2020 who were continuing on mepolizumab past 12 months, including: \\ medication use, exacerbation rate, asthma control questionnaire (ACQ), asthma quality of life questionnaire \\ (AQLQ), spirometry, fractional expired nitric oxide $\left(F_{\text {eNO }}\right)$, and sputum and blood eosinophil count. Data \\ were only included from participants consented to the Manchester Allergy, Respiratory and Thoracic \\ Surgery Biobank (MANARTS 87; M2020-87; IRAS107832). The accrual of complete datasets was \\ disrupted by the coronavirus disease pandemic from March 2020. \\ Statistical analysis was performed using SPSS software version 25 (IBM corporation, Chicago, IL, USA). \\ The majority of data were non-normally distributed and reported as median (interquartile range). \\ Between-group comparisons were made using Mann-Whitney U-test, and within-group longitudinal \\ changes investigated over 12 and 24 months using Wilcoxon and Friedman tests respectively. Categorical \\ data were compared using Chi-squared or Fisher's exact test. \\ 50 patients were included with age 52 (45-61) years, weight 87 (72-100) kg, 70\% female. Approval for \\ mepolizumab was based on maintenance prednisolone requirement (minimum $5 \mathrm{mg}$ per day) in 39 of 50 \\ patients, and minimum 3 (median 5 (interquartile range 4.5-7.5)) exacerbations in the preceding 12 months \\ in the other 11.15 patients (30\%) stopped mepolizumab during the second year of treatment (late failure \\ group) due to: patient choice $(n=2)$, side-effects $(n=5)$, and ongoing exacerbations and/or poor control with \\ persisting sputum eosinophilia $(n=8)$. Respiratory comorbidities were more common (Chi-squared \\ $\mathrm{p}=0.009)$ in late failure $(8 / 15)$ versus sustained responders $(6 / 35)$. \\ There were no differences in age, gender, weight or adherence between groups. Prior to starting \\ mepolizumab, sustained responders had lower blood eosinophils at approval (0.42 (0.35-0.55) versus 0.62 \\ $(0.4-0.72)$ cells $\left.\times 10^{9} \cdot \mathrm{L}^{-1} ; \mathrm{p}=0.017\right)$ and better ACQ (2.75 (1.81-4.04) versus $\left.3.83(2.95-5.08) ; \mathrm{p}=0.018\right)$ \\ than late failures. After 12 months of treatment, sustained responders had better ACQ (2.00 (0.83-3.51)

\section{○@®®} \\ Shareable abstract (@ERSpublications) \\ This study found that $30 \%$ of patients stopped mepolizumab in the second year of treatment. \\ Those with a sustained response had lower blood eosinophils and better asthma control \\ questionnaire scores prior to starting mepolizumab. https://bit.ly/3ChWN63 \\ Cite this article as: Elsey L, Pantin T, Holmes L-J, et al. Outcomes over the first two years of treatment \\ with mepolizumab in severe asthma. Eur Respir J 2021; 58: 2101313 [DOI: 10.1183/13993003.01313- \\ 2021].
}


versus 3.00 (2.41-4.40); $\mathrm{p}=0.016)$ and AQLQ (4.73 (3.80-6.20) versus 3.53 (3.06-4.73); $\mathrm{p}=0.012)$ than late failures.

Over the first 12 months of treatment, both groups had improvement in AQLQ, blood eosinophils and prednisolone dose, but only sustained responders decreased their exacerbation rate and ACQ (table 1).

In sustained responders, there was a further fall in daily prednisolone dose between months 12 and 24 from median (interquartile range) $5(0-6)$ to $0(0-5) \mathrm{mg}$ per day $(\mathrm{n}=35 ; \mathrm{p}=0.035)$, but no significant additional improvement in ACQ $(\mathrm{n}=33 ; 2.00(0.83-3.52)$ to $1.83(0.58-3.25))$, AQLQ ( $\mathrm{n}=25 ; 4.46$ (3.63$6.03)$ to $5.00(2.76-5.85))$, or annual exacerbation rate $(n=31 ; 1(0-1)$ to $1(0-3))$.

10 patients in the late failure group had sputum cell counts analysed during year 2; median sputum eosinophils were higher in this group $(6.0 \%(2.3-18.3 \%))$ compared to the eight sustained responders who had sputum analysed $(1.0 \%(0.0-1.9 \%) ; \mathrm{p}=0.045)$. Eight of the 10 late failures had raised sputum eosinophils $(>2 \%)$ compared to two of the eight sustained responders (Fisher's exact $\mathrm{p}=0.05$ ).

Almost a third of patients who had initially responded to mepolizumab at 12 months subsequently had treatment withdrawn by month 24. This group had a higher incidence of co-existent respiratory conditions, most commonly expiratory dynamic airways collapse, bronchiectasis, breathing pattern disorder or inducible laryngeal obstruction, and in over half there was sputum eosinophilia. In the context of persisting uncontrolled symptoms or exacerbations this represents a "treatable trait", although currently treatment options are limited. In both responders and late failures, the median ACQ was still over 1.5, and there was still a residual burden of exacerbations and OCS use, suggesting in many there was still a need for treatment optimisation.

The response in both groups over 12 months was similar to that seen in clinical trials and the Realiti-A study [7] with improvements in AQLQ, blood eosinophils and prednisolone exposure. Interestingly, only the sustained responders group improved their exacerbation rate and ACQ score. Those patients who went on to continue to respond up to 24 months had sustained improvements in asthma control and quality of life scores, demonstrating the importance of these objective measures in assessing patients' continued response to mepolizumab.

In the MENSA study [5] positive anti-mepolizumab antibodies were found in 19 patients, including 5\% of those in the subcutaneous mepolizumab group. This could potentially explain a proportion of our late failures. Currently a test for these antibodies is not available.

Patients with lower blood eosinophil counts at approval (and better asthma control at baseline) were more likely to have a sustained response with mepolizumab. Whilst in general higher blood eosinophils predict better response to anti-IL-5 biologics [8], it could be speculated that within cohorts pre-selected for eosinophilia those with especially high blood eosinophil counts (and worse asthma control) have more severe, and treatment-resistant, disease. On the other hand, baseline blood eosinophil counts, taken at the

TABLE 1 Clinical outcomes for sustained responders and late failures over the first 12 months of mepolizumab treatment

\begin{tabular}{|c|c|c|c|c|c|c|c|c|}
\hline & \multicolumn{4}{|c|}{ Sustained responders } & \multicolumn{4}{|c|}{ Late failures } \\
\hline & $\mathrm{n}$ & Baseline & 12 months & $\mathrm{p}$-value & $\mathrm{n}$ & Baseline & 12 months & $\mathrm{p}$-value \\
\hline$A C Q$ & 34 & $2.75(1.81-4.04)$ & $2.00(0.83-3.51)$ & 0.003 & 13 & $3.83(2.95-5.08)$ & $3.00(2.41-4.40)$ & 0.173 \\
\hline AQLQ & 31 & $3.40(2.20-4.06)$ & $4.73(3.80-6.20)$ & $<0.001$ & 11 & $2.07(1.70-3.80)$ & $3.53(3.06-4.73)$ & 0.033 \\
\hline FEV $_{1} \%$ pred & 32 & $73.5(64.5-85.2)$ & $78.5(64.0-95.3)$ & 0.651 & 10 & $77.0(59.5-87.3)$ & $67.5(57.5-84.3)$ & 0.168 \\
\hline$F_{\mathrm{eNO}} \mathrm{ppb}$ & 28 & $21.0(10.3-36.5)$ & $24.0(9.0-40.8)$ & 0.476 & 12 & $34.5(16.0-58.3)$ & $29(16.8-85.3)$ & 0.505 \\
\hline Blood eosinophils $\times 10^{9}$ cells $\cdot \mathrm{L}^{-1}$ & 34 & $0.20(0.07-0.33)$ & $0.04(0.02-0.07)$ & $<0.001$ & 10 & $0.26(0.11-0.59)$ & $0.06(0.03-0.13)$ & 0.008 \\
\hline Exacerbations per year & 26 & $4.0(0.0-5.3)$ & $1.0(0.0-1.3)$ & 0.006 & 8 & $1.0(0.0-4.0)$ & $1.5(0.3-2.8)$ & 0.799 \\
\hline Prednisolone dose $\mathrm{mg}$ per day & 35 & $10(5-20)$ & $5(0-6)$ & $<0.001$ & 13 & $10(3-20)$ & $4(1-11)$ & 0.008 \\
\hline Adherence $\%$ pickup & 22 & $80(80-100)$ & $90(79-100)$ & 0.876 & 10 & $80(80-85)$ & $80(80-100)$ & 0.705 \\
\hline
\end{tabular}

Data are presented as median (interquartile range), unless otherwise stated. Significance level ( $\mathrm{p}$-value) in change versus baseline by Wilcoxon signed rank test. ACQ: asthma control questionnaire; AQLQ: asthma quality of life questionnaire; $\mathrm{FEV}_{1}$ : forced expiratory volume in $1 \mathrm{~s}$; $F_{\mathrm{eNO}}$ : fractional expired nitric oxide. Baseline blood eosinophils in the table were measured at initiation of treatment, and therefore were not the same as those in the decision for approval of biologic therapy. 
initiation of treatment, were not different between groups. This disparity could be explained by the use of increased corticosteroid doses to stabilise disease between approval and initiation, or alternatively it could be a spurious finding.

Aerosol-generating procedures could not be performed after March 2020 due to the coronavirus pandemic; this led to some missing lung function and $F_{\text {eNO }}$ data at 24 months. Whilst there is no evidence to our knowledge that the pandemic has led to adverse outcomes in severe asthma, and none of our patients reported infection with COVID-19, it is theoretically possible that some of the "late failures" may have been due to the infection.

In conclusion, we have found a significant minority of patients with severe asthma responding to mepolizumab over the first 12 months of therapy subsequently withdraw treatment in the following year. Although sustained responders achieve further reductions in prednisolone exposure, improvements in control and quality of life may plateau at a "new-normal" rather than continue to improve. These findings support continued review of treatment on at least a 12-monthly basis, and consideration of further investigation where improvements are unsatisfactory. Further studies are needed to investigate treatment options in such patients.

Lynn Elsey $\oplus^{1}$, Thomas Pantin $\oplus^{1}$, Leanne-Jo Holmes ${ }^{1}$, Gael Tavernier $\oplus^{1,2}$ and Stephen J. Fowler $\oplus^{1,2}$

${ }^{1}$ North West Lung Centre, Manchester University NHS Foundation Trust, Manchester, UK. ${ }^{2}$ Faculty of Biology, School of Biological Sciences, Medicine and Health, University of Manchester, Manchester Academic Health Science Centre and NIHR Manchester Biomedical Research Centre, Manchester University Hospitals NHS Foundation Trust, Manchester, UK.

Corresponding author: Lynn Elsey (lynn.elsey@mft.nhs.uk)

Acknowledgements: We would like to thank the staff of the severe asthma nursing team and Philippa Aspin, research scientist, for assistance with data collection. We acknowledge the support of the NIHR Manchester Clinical Research Facility, Manchester Allergy, Respiratory and Thoracic Surgery (ManARTS) Biobank in carrying out this study. S.J. Fowler is supported by the NIHR Manchester Biomedical Research Centre.

Conflict of interest: L. Elsey and L-J. Holmes report payments for presentations, advisory boards and speaker fees from AstraZeneca, GSK, Teva, Chiesi, Sanofi and Novartis; support to meetings from AstraZeneca, Teva, GSK and Novartis; outside the submitted work. T. Pantin reports support to attend meetings from Chiesi, Sanofi and GSK; outside the submitted work. G. Tavernier has nothing to disclose. S.J. Fowler reports grants from Boehringer Ingelheim; speaker fees from Boehringer Ingelheim, AstraZeneca, Teva, Chiesi, GSK and Novartis; support to attend meetings from Chiesi, Sanofi, GSK and AstraZeneca.

\section{References}

1 Global Initiative for Asthma. Difficult to Treat and Severe Asthma in Adolescent and Adult Patients. Diagnosis and Management. Global Initiative for Asthma, 2019.

2 National Institute for Health and Care Excellence. Mepolizumab for Treating Severe Refractory Eosinophilic Asthma [TA671]. www.nice.org.uk/guidance/ta671 Date last accessed: 1 March 2021. Date last updated: 3 Feb 2021.

3 Bel EH, Wenzel SE, Thompson PJ, et al. Oral glucocorticoid-sparing effect of mepolizumab in eosinophilic asthma. N Engl J Med 2014; 371: 1189-1197.

4 Chupp GL, Bradford ES, Albers FC, et al. Efficacy of mepolizumab add-on therapy on health-related quality of life and markers of asthma control in severe eosinophilic asthma (MUSCA): a randomised, double-blind, placebo-controlled, parallel-group, multicentre, phase 3b trial. Lancet Respir Med 2017; 5: 390-400.

5 Ortega HG, Liu MC, Pavord ID, et al. Mepolizumab treatment in patients with severe eosinophilic asthma. N Engl J Med 2014; 371: 1198-1207.

6 Pavord ID, Korn S, Howarth P, et al. Mepolizumab for severe eosinophilic asthma (DREAM): a multicentre, double-blind, placebo-controlled trial. Lancet 2012; 380: 651-659.

7 Harrison T, Canonica GW, Chupp G, et al. Real-world mepolizumab in the prospective severe asthma REALITI-A study: initial analysis. Eur Respir J 2020; 56: 2000151.

8 Kroes JA, Zielhuis SW, van Roon EN, et al. Prediction of response to biological treatment with monoclonal antibodies in severe asthma. Biochem Pharmacol 2020; 179: 113978. 